\title{
Screen-based identification and validation of four new ion channels as regulators of renal ciliogenesis
}

\author{
Gisela G. Slaats ${ }^{1, *}$, Gabrielle Wheway ${ }^{2, *}$, Veronica Foletto ${ }^{1}$, Katarzyna Szymanska ${ }^{2}$, Bas W. M. van Balkom ${ }^{1}$, \\ Ive Logister ${ }^{1}$, Krista Den Ouden ${ }^{1}$, Mandy G. Keijzer-Veen ${ }^{3}$, Marc R. Lilien ${ }^{3}$, Nine V. Knoers ${ }^{4}$, Colin A. Johnson ${ }^{2}$ \\ and Rachel H. Giles ${ }^{1, \pm}$
}

\begin{abstract}
To investigate the contribution of ion channels to ciliogenesis, we carried out a small interfering RNA (siRNA)-based reverse genetics screen of all ion channels in the mouse genome in murine inner medullary collecting duct kidney cells. This screen revealed four candidate ion channel genes: Kcnq1, Kcnj10, Kcnf1 and Clcn4. We show that these four ion channels localize to renal tubules, specifically to the base of primary cilia. We report that human KCNQ1 Long QT syndrome disease alleles regulate renal ciliogenesis; KCNQ1-p. R518X, -p.A178T and -p.K362R could not rescue ciliogenesis after Kcnq1-siRNA-mediated depletion in contrast to wild-type KCNQ1 and benign KCNQ1-p.R518Q, suggesting that the ion channel function of $\mathrm{KCNQ} 1$ regulates ciliogenesis. In contrast, we demonstrate that the ion channel function of KCNJ10 is independent of its effect on ciliogenesis. Our data suggest that these four ion channels regulate renal ciliogenesis through the periciliary diffusion barrier or the ciliary pocket, with potential implication as genetic contributors to ciliopathy pathophysiology. The new functional roles of a subset of ion channels provide new insights into the disease pathogenesis of channelopathies, which might suggest future therapeutic approaches.
\end{abstract}

KEY WORDS: Cilia, siRNA screen, Patient mutation, KCNQ1, KCNJ10, KCNF1, CLCN4

\section{INTRODUCTION}

Primary cilia, also referred to as immotile or sensory cilia, project from most cells and function to sense their anatomical and/or physiological environment (Satir and Christensen, 2008). In general, primary cilia transduce signals from extracellular stimuli to a cellular response that regulates proliferation, differentiation, transcription, migration, polarity and tissue morphology (Oh and Katsanis, 2012) in a tissue-specific manner (D’Angelo and Franco, 2011). Developmental and pathological pathways of cellular signaling linked to primary cilia include, but are not limited to, canonical and non-canonical Wnt, Hedgehog and Hippo signaling (Basten and Giles, 2013).

Primary cilia play an important role in the development and healthy physiology of many different organs, including eye, kidney, liver, brain and bone. Reflecting the complexity of ciliogenesis and

\footnotetext{
${ }^{1}$ Department of Nephrology and Hypertension, University Medical Center Utrecht, Utrecht 3584 CX, The Netherlands. ${ }^{2}$ Section of Ophthalmology and Neuroscience, Leeds Institutes of Molecular Medicine, University of Leeds, Leeds LS9 7TF, UK. ${ }^{3}$ Department of Pediatric Nephrology, University Medical Center Utrecht, Utrecht 3584 CX, The Netherlands. 'Department of Medical Genetics, University Medical Center Utrecht, Utrecht 3584 CX, The Netherlands.

*These authors contributed equally to this work

${ }^{\ddagger}$ Author for correspondence (r.giles@umcutrecht.nl)
}

Received 19 June 2015; Accepted 4 November 2015 the essential role of primary cilia in signal transduction in a large variety of tissues and organ systems, numerous diseases have been linked to abnormal cilium function (Baker and Beales, 2009). Of the so-called ciliopathies, kidney cysts are one of the more frequent phenotypes (Baker and Beales, 2009), and polycystic kidney diseases collectively represent one of the most common genetic diseases known to mankind (Wilson, 2004). Ion channels also have crucial roles in the renal epithelium, regulating $\mathrm{Na}^{+}, \mathrm{K}^{+}$, and $\mathrm{Mg}^{2+}$ homeostasis, as well as the control of water reabsorption in the collecting duct (Loudon and Fry, 2014). To study the roles of ion channels further, we systematically interrogated the contribution of all ion channels to ciliogenesis or maintenance of primary cilia in collecting duct cells of the kidney.

We designed a small interfering RNA (siRNA)-based reverse genetics screen of all ion channels in the mouse genome in murine inner medullary collecting duct (mIMCD3) cells that identified effects on ciliary structure (ciliogenesis) instead of function (signaling). This revealed four ion channel genes with a significant effect on ciliogenesis, but not on cell number: Kcnql $\left(\mathrm{K}^{+}\right.$channel, voltage-gated, kqt-like subfamily, member 1), Kcnj10 (potassium channel, inwardly rectifying, subfamily J, member 10), Kcnfl ( $\mathrm{K}^{+}$channel, voltage-gated, subfamily F, member 1) and Clcn4 $\left(\mathrm{Cl}^{-}\right.$channel, voltage-gated 4). We took all of these candidates forward for validation and further functional analysis, with human disease alleles of $K C N J 10$ and $K C N Q 1$ tested in 3D spheroids to examine their effects on ciliogenesis. We also observed that all four endogenous proteins localize to membrane subdomains at the base of primary cilia. We conclude that the localization of these channels to membrane subdomains at the base of the cilium, which have been previously identified to regulate ciliogenesis, reflects an influence on membrane function or vesicle transport adjacent to the transition zone or at the ciliary pocket. By elucidating the role of these ion channels in primary cilia and disease, we offer new insights into the disease pathogenesis of channelopathies and opportunities for potential improvements to disease management.

\section{RESULTS}

\section{Reverse genetics screen}

To investigate the potential roles of ion channels on renal ciliogenesis, we performed an siRNA-based reverse genetics screen of all ion channels in the mouse genome in mouse inner medullary collecting duct cells (mIMCD3 cells). This screen represents 340 ion channels across the mouse genome (Table S1). The effect of siRNA knockdown on ciliogenesis was assessed by calculating the percentage of cells with a single cilium, with the statistical significance of this effect assessed by calculating $z$ scores $\left(z_{\text {cilia }}\right) . z$ scores were also calculated for the effect of the knockdown on cell number $\left(z_{\text {cell }}\right)$, to exclude effects of cell proliferation or apoptosis on ciliogenesis, because these could be due to non- 


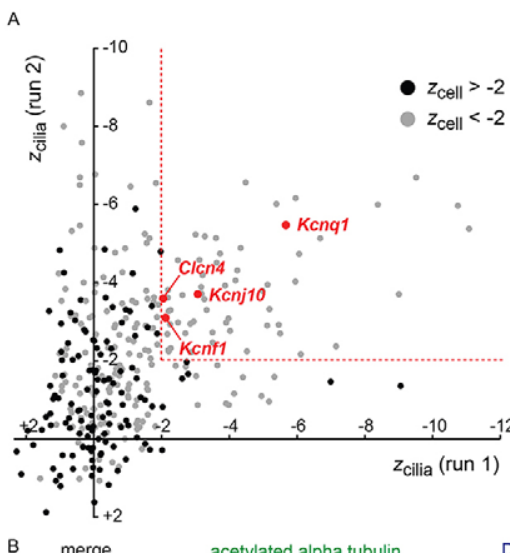

B merge acetylated alpha tubulin DAPI

Kcnq1 plate 1 well A3 FOV 4
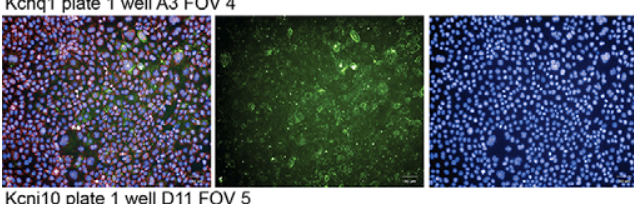

TOTO3
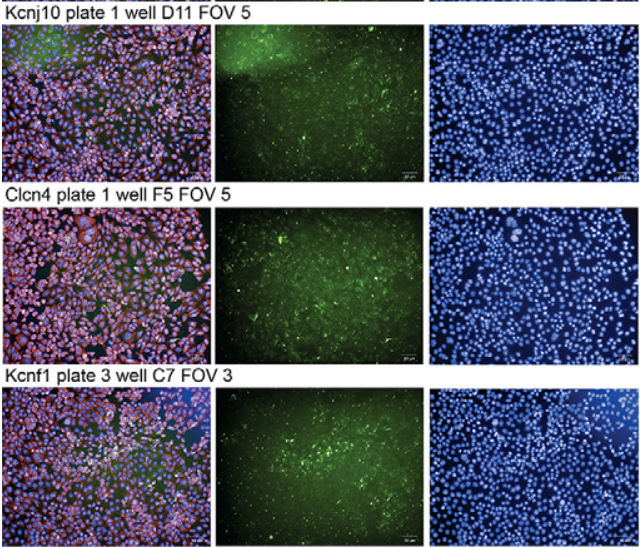

scrambled non-targeting siRNA plate 1 well G1 FOV 6

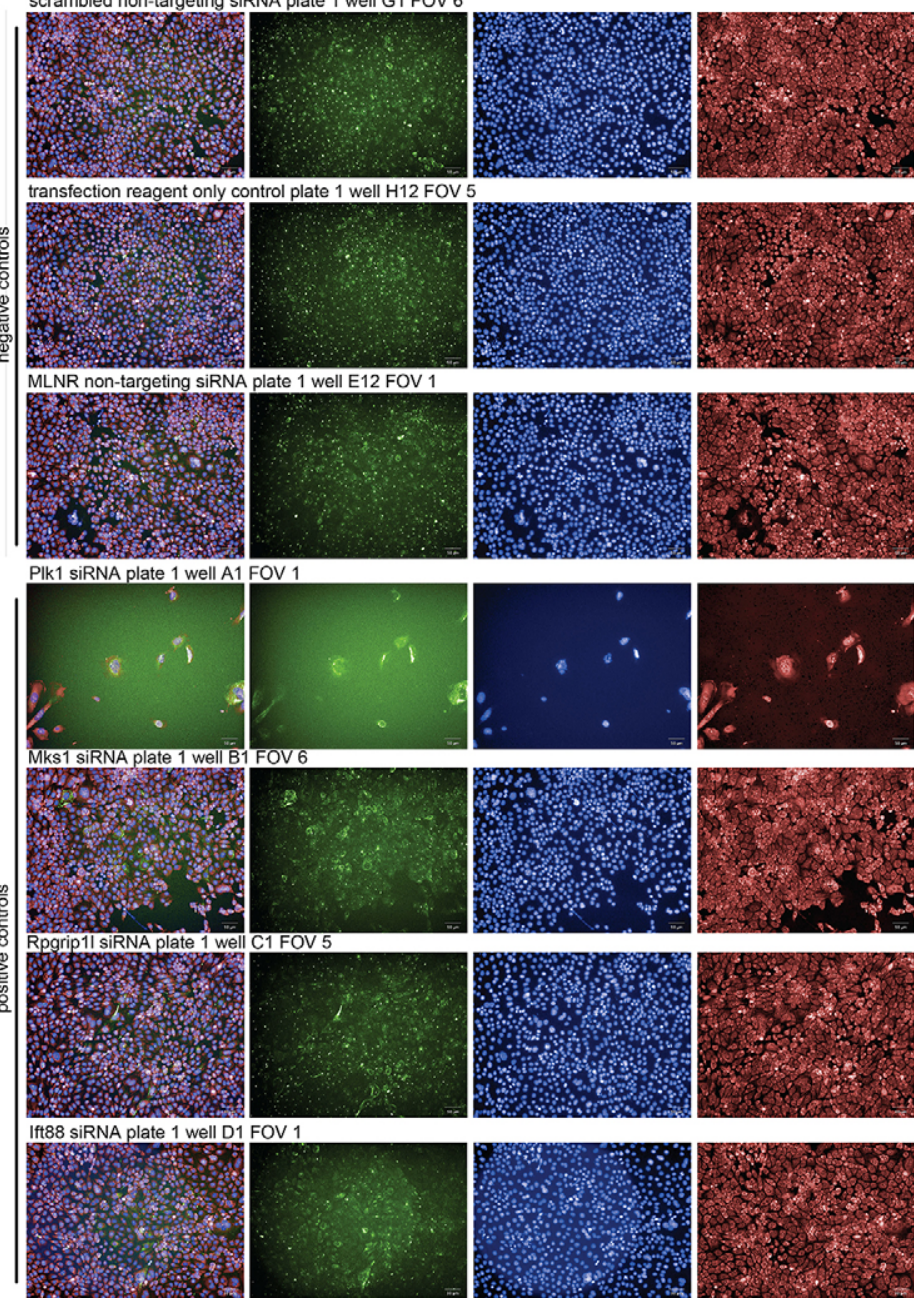

Fig. 1. Scatter-plot and images of the siRNA-based ion channels screen. (A) Scatter-plot of $z$-scores from both runs of the siRNA screen. The cut-offs of $z_{\text {cilia }}$ at $<-2$ for both run 1 and run 2 are shown with dotted red lines. Gray and black points discriminate between $z_{\text {cell }}>-2$ or $<-2$ (gray means exclusion).

(B) Representative images of the negative controls, the positive controls and the four ion channel candidate genes. Immunostaining of mIMCD3 cells for cilia (acetylated $\alpha$-tubulin, green) and TOTO3 (red), with DAPI counterstaining (blue) shows loss of cilia after Ift88, Kcnj10, Kcnq1, Kcnf1 and Clcn4 siRNA transfection (50 nM).

specific secondary processes (Wheway et al., 2015) (Fig. 1). Based on this analysis, we identified four ion channels that had a significant negative effect on ciliogenesis but not on cell number: Kcnq1, Kcnj10, Kcnf1 and Clcn4 (Table S1; Fig. 1).

\section{Validation screening - all four ion channels localize to the primary cilium and are required for ciliogenesis}

To validate the effect of knockdowns of Clcn4, Kcnfl, Kcnql and Kcnj10 on ciliogenesis we repeated the siRNA knockdowns in mIMCD3 cells, followed by immunofluorescence staining of cilia using an antibody against acetylated $\alpha$-tubulin and analysis by confocal microscopy. The ciliary marker ARL13B always colocalized with acetylated $\alpha$-tubulin in IMCD3 cells, justifying the use of acetylated $\alpha$-tubulin as a cilia marker in our experiments (Fig. S1A). We tested ON-TARGETplus SMARTpool siRNA knockdown efficiency of Clcn4, Kcnfl, Kcnq1 and Kcnj10 at the mRNA level by quantitative real-time PCR (RT-qPCR), and found all siRNAs to reduce transcript levels compared to cells transfected with non-targeting control siRNA (siControl) (Fig. S3A). Knockdown of each of the four genes resulted in a lower percentage of ciliated cells compared to cells transfected with
siControl (Fig. 2A; Fig. S1B). Additionally, we tested the effect of siRNA-mediated depletion of Clcn4, Kcnfl, Kcnql and Kcnj10 on ciliation in 3D spheroids, a commonly used model for renal ciliopathies (Giles et al., 2014). 3D spheroids transfected with siRNAs to deplete cellular levels of any of these four ion channels manifested significantly lower cilia frequency compared to nontargeting siRNA-treated control cells. The reduction in the cilia levels was comparable to that achieved with Ift88 siRNA knockdown, a well-accepted regulator of ciliogenesis used here as a positive control (Pazour et al., 2000; Wheway et al., 2015) (Fig. 2B). Cell cycle phase distribution analysis showed unchanged cell numbers in the G1-, S- and G2-phase of the cell cycle after siRNA-mediated depletion of Clcn4, Kcnq1 and Kcnj10 compared to siControl, excluding any possible cell cycle effect on the observed loss of cilia (Fig. S3B).

Immunofluorescence staining using specific antibodies to each of these ion channels showed that endogenous Clcn4, Kcnf1, Kcnq1 and Kcnj10 proteins localize to membrane subdomains at the base of primary cilia of human fibroblasts and mIMCD3 cells (Fig. 2C; Fig. S2), in addition to the previously described localizations of these channels at the endosomal membrane for CLCN4 (Scheel 
A

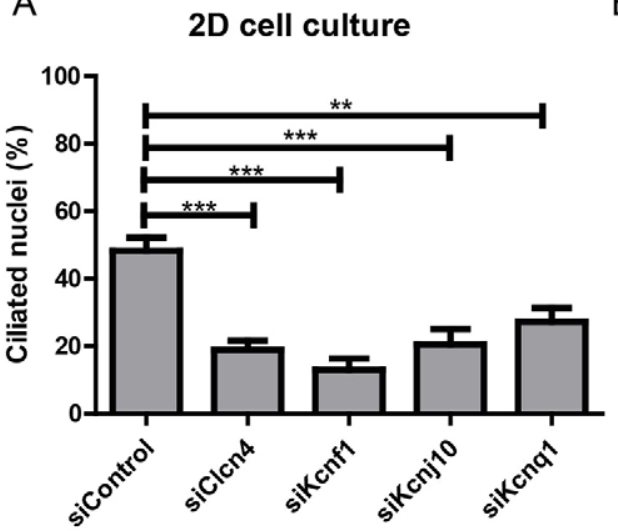

C

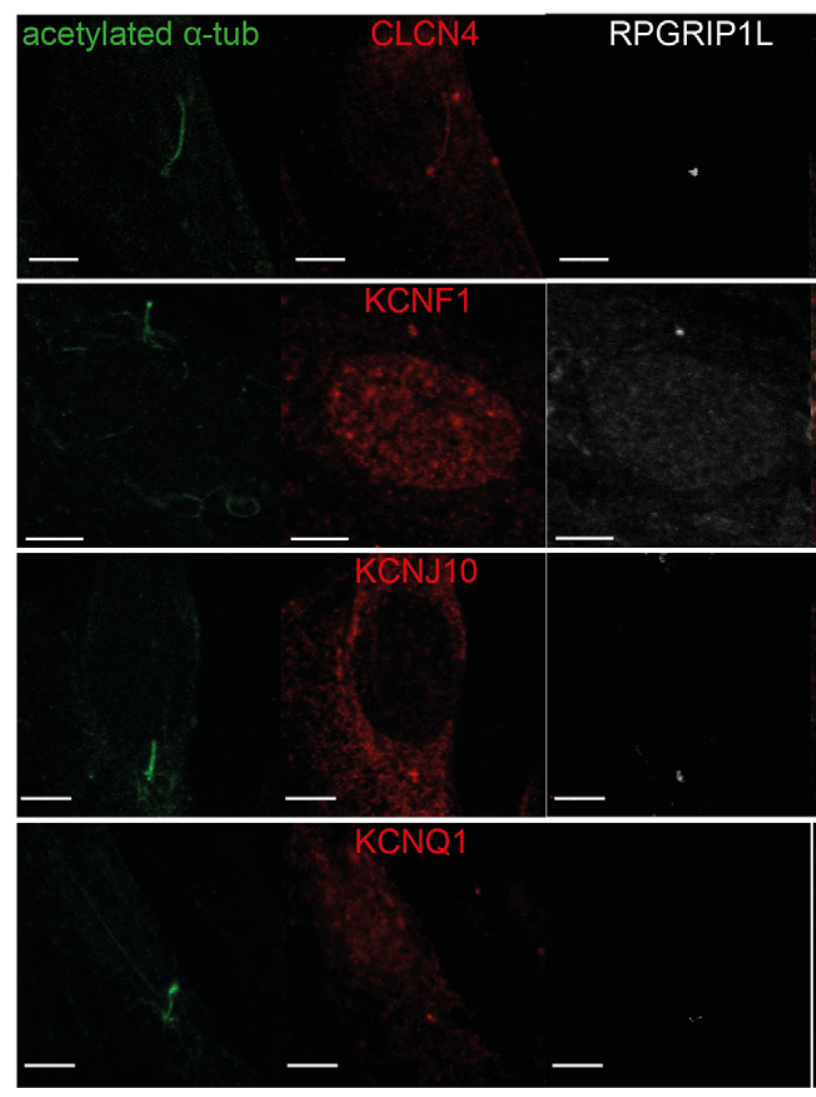

et al., 2005), or basolateral membrane for KCNQ1 and KCNJ10 (Jespersen et al., 2004; Reichold et al., 2010). In human fibroblasts derived from two unrelated individuals, CLCN4 decorates the entire length of the cilium (Fig. 2C), whereas in murine renal cells it is restricted to the membrane subdomain at the base of the cilium, suggesting species- and tissue-specificity of localization. Additionally, histology of rat paraffin-embedded kidney sections demonstrates localization of the ion channels to renal tubules (Fig. S4). Furthermore, we examined overexpression of wild-type (WT) human CLCN4-GFP, KCNJ10-GFP and KCNQ1 in 3D spheroids of mIMCD3 cells (Giles et al., 2014). CLCN4-GFP and KCNJ10-GFP protein localized to primary cilia (white arrows) (Fig. 3A), validating localization of endogenous ion channels observed at primary cilia (Fig. 2C; Fig. S2). Overexpression of the human WT protein in 3D spheroids rescued loss of cilia after siRNA knockdown of endogenous Clcn 4, Kcnj10 and Kcnql (Fig. 3B). In addition to GFP-tagged overexpression of WT CLCN4 and
Fig. 2. CLCN4, KCNF1, KCNJ10 and KCNQ1 localize to primary cilia and regulate

primary cilia. (A) Quantification (mean \pm s.e.m., $\%$ ) of ciliated mIMCD3 cells shows loss of cilia after siRNA-mediated depletion $(20 \mathrm{nM})$ for Clcn4, Kcnf1, Kcnj10 or Kcnq1. >250 cells were scored per condition $(n=3) .{ }^{* *} P<0.01$, ${ }^{* * *} P<0.001$ (one-way ANOVA with Dunnett's test). (B) Quantification (mean \pm s.e.m., \%) of cilia in 3D spheroids shows loss of cilia in spheroids depleted for Clcn4, Kcnf1, Kcnj10, Kcnq1 or Ift88. 25 spheroids were scored per condition. ${ }^{*} P<0.05,{ }^{*} P<0.01$ and ${ }^{* * *} P<0.001$ $n=3$ (one-way ANOVA with Dunnett's test).

(C) Immunostaining of human fibroblasts after serum starvation for cilia (acetylated $\alpha$-tubulin, green), the transition zone (RPGRIP1L, white) and the different ion channels CLCN4, KCNF1, KCNJ10 and KCNQ1 (red) with DAPI

counterstaining (blue) shows localization of all four ion channels to primary cilia (magnified inserts). Scale bars: $5 \mu \mathrm{m}$.
KCNJ10 (Fig. 3A), western blot confirmed overexpression of WT KCNJ10 (Fig. S3C) and KCNQ1 (Fig. S3D).

As human mutations have been identified in $K C N Q 1$ and $K C N J 10$ patients with inherited disease that have some features overlapping with ciliopathy phenotypes, we further tested whether patient mutations might affect the ability of the overexpressed proteins to rescue the ciliogenesis defect after Kcnq1 and Kcnj10 knockdown.

\section{KCNJ10 EAST syndrome alleles}

Mutations in $K C N J 10$ have been associated with epilepsy, ataxia, sensorineural deafness and (Gitelman-like) tubulopathy (EAST syndrome, alternatively named SESAME syndrome), an autosomal recessive disease (MIM 612780) occasionally featuring seizures and mental retardation. To test the effect of EAST-syndromeassociated human mutations on KCNJ10 function in primary cilia, we examined the ability of WT and two mutant $K C N J 10$ alleles to rescue the ciliogenesis defect seen in 3D spheroids after Kcnj10 

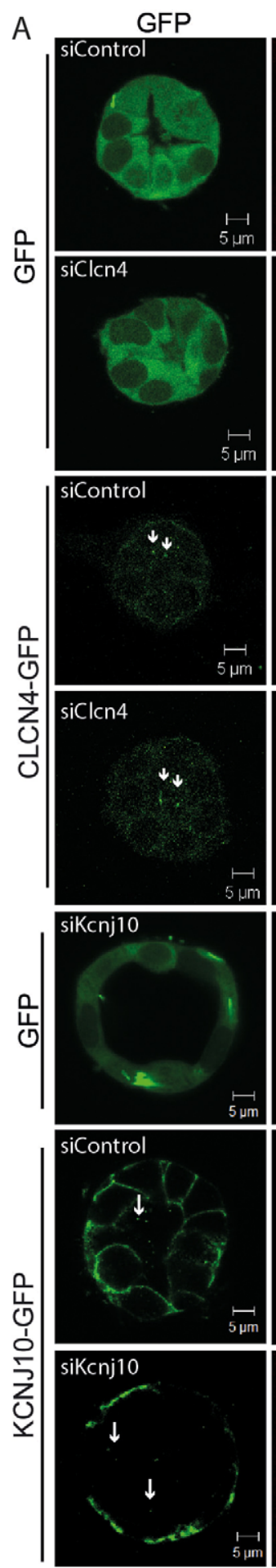

Ac. Tubulin
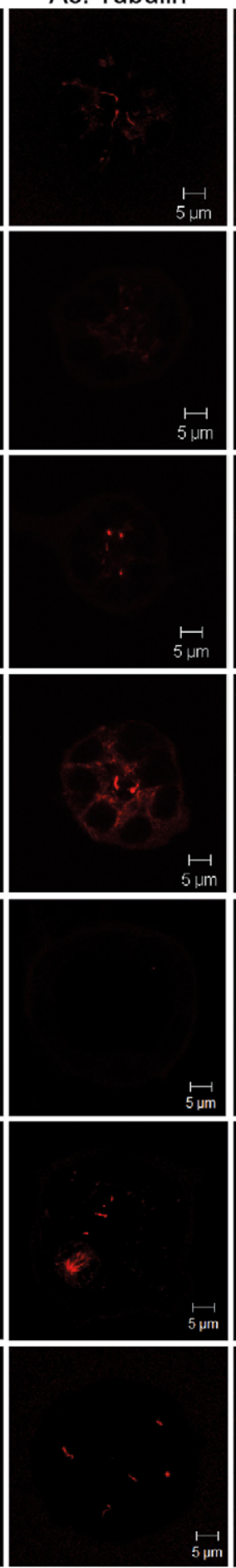

Merged
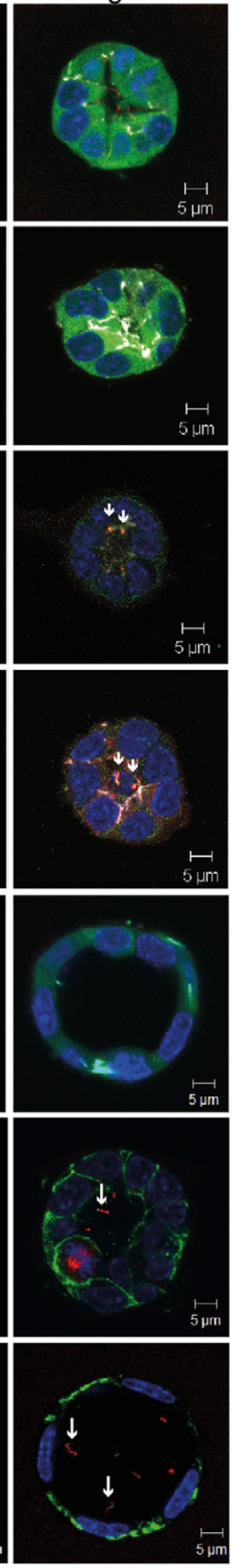
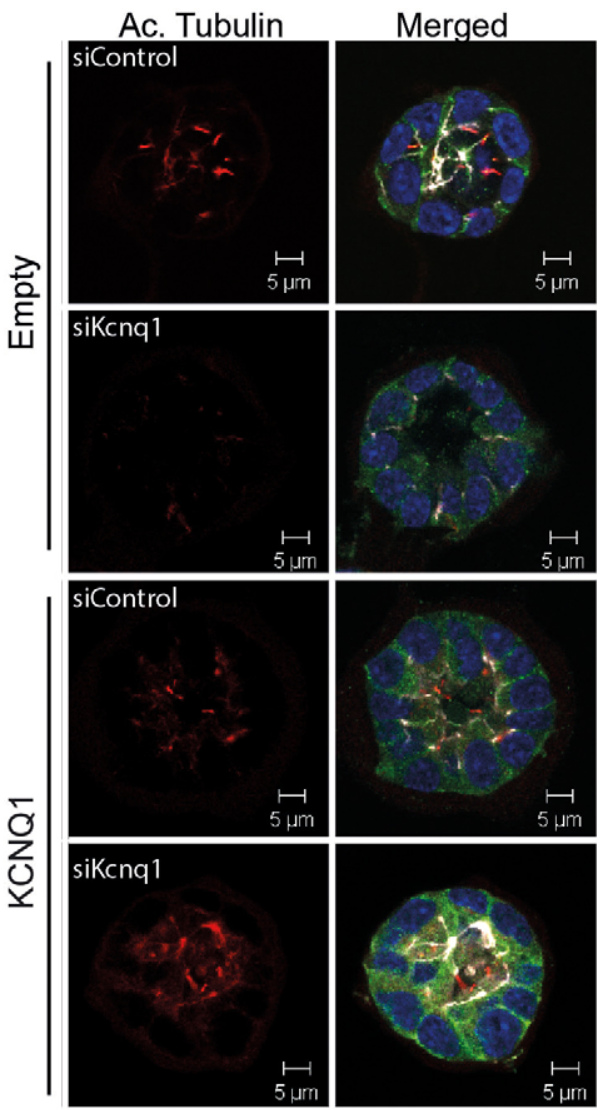

B
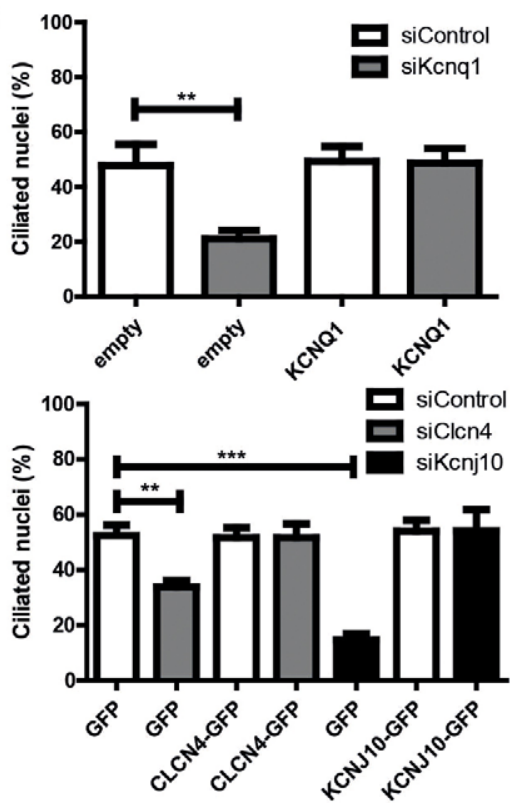

Fig. 3. Ciliation in 3D spheroids with wild-type CLCN4-GFP, KCNJ10-GFP and KCNQ1. (A) Immunostaining of spheroids of mIMCD3 cells for cilia (acetylated $\alpha$-tubulin, red), tight junctions (ZO-1, white), with DAPI counterstaining (blue) shows loss of cilia after Clcn4, Kcnj10 and Kcnq1 siRNA transfection (20 nM), and rescue of ciliation by overexpression of wild-type CLCN4-GFP, KCNJ10-GPF or KCNQ1. White arrows indicate localization of CLCN4-GFP and KCNJ10GFP to primary cilia. In KCNQ1 spheroids, adherens junctions ( $\beta$-catenin, green) are also stained. (B) Quantification (mean \pm s.e.m., \%) of ciliary frequency in spheroids shows loss of cilia in spheroids depleted for Kcnq1, Clcn4), and Kcnj10, and restoration of ciliary frequency was obtained upon transfection with wildtype KCNQ1, CLCN4-GFP, and KCNJ10-GFP, respectively. 20 spheroids were scored per condition, $n=3 .{ }^{* \star} P<0.01$, ${ }^{* \star *} P<0.001$ (two-way ANOVA with Bonferroni corrections).

siRNA knockdown. A previous study performed patch clamp assays on a series of KCNJ10 mutant alleles to quantify the effect of the missense mutation on ion channel function, showing many of them to be hypomorphic (Parrock et al., 2013). The alleles we tested were:
KCNJ10-c.194G >C/p.R65P (20\% retained function) and KCNJ10c. $418 \mathrm{~T}>\mathrm{C} / \mathrm{p} . \mathrm{C} 140 \mathrm{R}$ (total loss of function), normalized to KCNJ10WT (100\% function). All KCNJ10 alleles were transfected into mIMCD3 cells and, $24 \mathrm{~h}$ later, cells were transfected with non- 

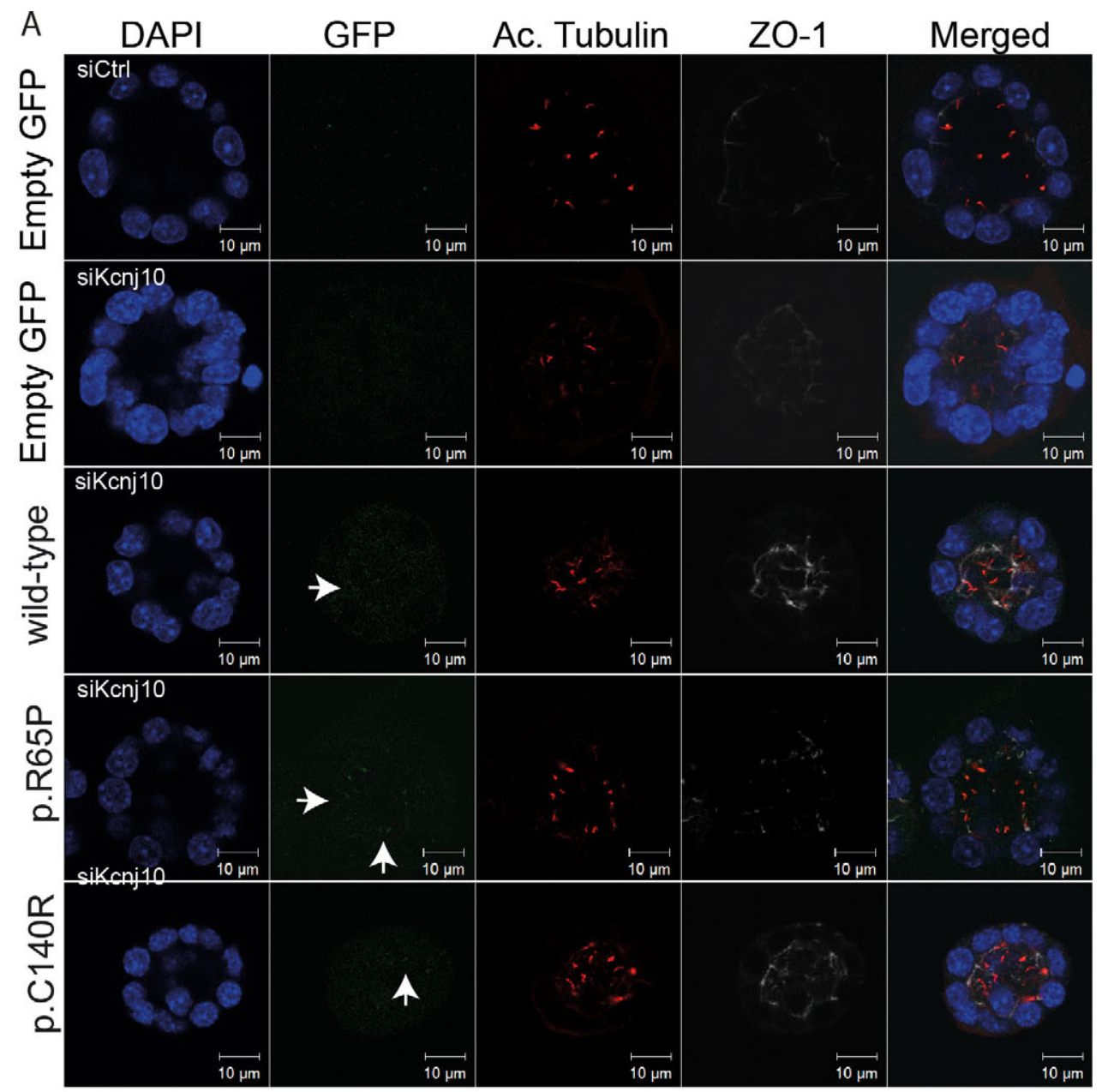

B

siControl 3D spheroids

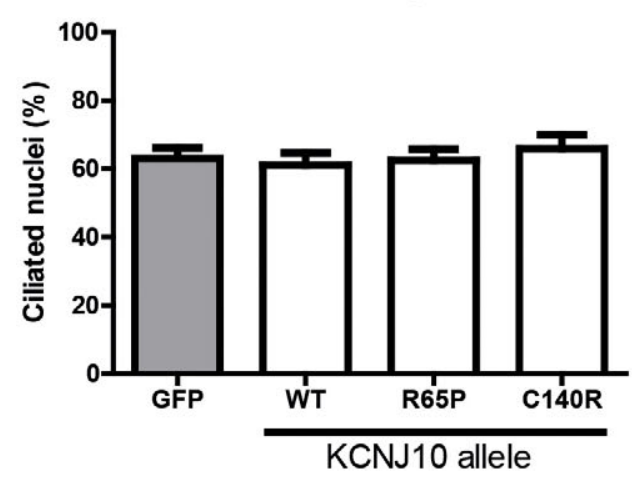

C

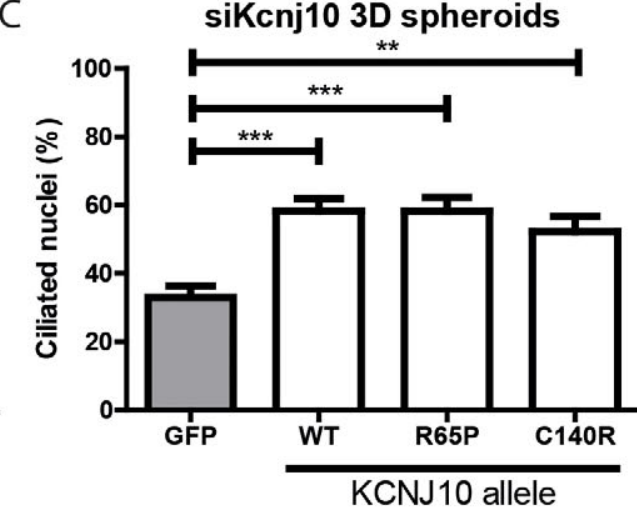

Fig. 4. 3D spheroids of KCNJ10 EAST syndrome-associated alleles.

(A) Immunostaining of spheroids of mIMCD3 cells for cilia (acetylated $\alpha$-tubulin red) and tight junctions (ZO-1, white), with DAPI counterstaining (blue) showing loss of cilia after Kcnj10 siRNA transfection (20 nM), and rescue of ciliation by wild-type (WT) and p.R65P and p.C140R

KCNJ10-GFP (white arrows indicate localization at cilia). (B,C) Quantification (mean \pm s.e.m., \%) of ciliary frequency in spheroids shows loss of cilia in spheroids depleted for Kcnj10, and restoration of ciliary frequency was obtained upon transfection with WT KCNJ10. Overexpression of variant KCNJ10 did not affect ciliation in siControltransfected cells. In siKcnj10-transfected cells, overexpression of KCNJ10 p.R65P and p.C140R also rescued ciliation. 20 spheroids were scored per condition $(n=3)$. ${ }^{* *} P<0.01,{ }^{* *} P<0.001$ (one-way ANOVA with Dunnett's test). targeting control siRNA or Kcnj10 siRNAs that did not target the human KCNJ10 constructs. KCNJ10-WT rescued loss of primary cilia after Kcnj10 knockdown to the same level as KCNJ10-p.R65P and p.C140R (Fig. 4A,C), indicating completely retained function of these alleles with regard to ciliogenesis. In addition, similar to the WT protein, both mutated proteins localized to the cilium (white arrows; Fig. 4A). No differences were found in cells transfected with any KCNJ10 construct and control siRNA (Fig. 4B). An assessment of the expression of the two missense mutant proteins, by western blotting, validated expression of all constructs (70 kDa; Fig. S3C). We conclude that loss of function of KCNJ10 is unlikely to affect primary cilia of EAST syndrome patients, and that the ciliary and ion channel functions of KCNJ10 are independent of each other.

\section{KCNQ1 alleles associated with Long QT syndrome}

Genetic variants of $K C N Q 1$ are known to cause Long QT syndrome (LQTS; MIM 192500), an inherited disease predominantly affecting heart function, as the KCNQ1 ion channel is required for the normal repolarization stage of the cardiac action potential (Sanguinetti et al., 1996; Wang et al., 1996). The autosomal dominant form of the condition is also known as Romano-Ward syndrome (MIM 192500). The autosomal recessive variant is known as Jervell and Lange-Nielsen syndrome (JLNS; MIM 220400), with congenital sensorineural hearing loss as an additional phenotypic feature (Neyroud et al., 1997). KCNQ1 variants are also associated with familial atrial fibrillation 3 (MIM 607554) (Chen et al., 2003), Short QT syndrome 2 (MIM 609621) (Bellocq et al., 
2004) and imprinting defects of KCNQ1 results in BeckwithWiedemann syndrome, a congenital overgrowth syndrome that often involves kidney abnormalities (MIM 130650) (Lee et al., 1997). Furthermore, renal patients might develop acquired LQTS, which is enhanced by $\mathrm{K}^{+}$-channel-inhibiting medication for their kidney disease (Gussak and Gussak, 2007).

To further investigate the potential role of KCNQ1 in renal primary cilia formation, we examined whether patient alleles associated with LQTS perturb ciliogenesis in renal cells. We generated expression plasmids engineered with human variants associated with the LQTS variants KCNQ1-c.1553G>A/p.R518Q (a benign variant) (Kapplinger et al., 2009), truncating allele KCNQ1-c.1552C > T/p.R518X (Stattin et al., 2012), KCNQ1c.532G >A/p.A178T (Tanaka et al., 1997; Kapplinger et al., 2009; Refsgaard et al., 2012) and KCNQ1-c.1085A>G/p.K362R (also associated with JLNS) (Tester et al., 2005; Kapplinger et al., 2009). All KCNQ1 alleles were transfected into mIMCD3 cells and, $24 \mathrm{~h}$ later, cells were transfected with non-targeting control siRNA or Kcnq1 siRNA (not targeting human KCNQ1). Both KCNQ1-WT and KCNQ1-p.R518Q rescued loss of primary cilia after Kcnq1 knockdown (Fig. 5A,C), indicating retained function of these alleles. In contrast, -p.R518X, -p.A178T and -p.K362R did not rescue the loss of cilia phenotype after Kcnql knockdown, indicating that these alleles might play a pathogenic role in the kidney of human patients (Fig. 5A,C). No differences were found in cells transfected with any KCNQ1 constructs and control siRNA, excluding a role for dominant-negative action (Fig. 5B). Additionally, we observed increased aberrant mitotic spindle orientation deviating $>11^{\circ}$ from the perpendicular radius or spindle pole plane (Luijten et al., 2013) in cells depleted of Kcnq1 compared to siControl-transfected cells (Fig. 5A, white arrows). Overexpression of WT or mutant KCNQ1-p.R518Q partly rescues aberrant mitotic spindle orientation, but, KCNQ1 alleles -p.R518X, -p.A178T and -p.K362R did not rescue spindle orientation (Fig. 5D). Aberrant mitotic spindle orientation could possibly be caused by polarization defects (Luijten et al., 2013). Western blot of overexpression of KCNQ1 and mutant alleles show expression of all constructs at $70 \mathrm{kDa}$, except for the truncated allele KCNQ1-p.R518X (predicted $\sim 55 \mathrm{kDa}$ ). KCNQ1-p.A178T has lower expression levels, which might explain why rescue is not observed following overexpression of this mutant (Fig. S3D).

\section{DISCUSSION}

We describe an unbiased siRNA knockdown screen of ion channels in a mouse ciliated cell line, and identified three $\mathrm{K}^{+}$and one $\mathrm{Cl}^{-}$ channel that are implicated in ciliogenesis without affecting cell number. Further work shows for the first time that these ion channels localize to the base of renal primary cilia, either to the diffusion barrier adjacent to the transition zone regulated by septin 2 (Hu et al., 2010), or to the ciliary pocket, which is an invaginated membrane at the base of the cilium characterized by enhanced endocytosis and vesicle trafficking (Lu et al., 2015). These four ion channels are required for ciliogenesis in $2 \mathrm{D}$ and $3 \mathrm{D}$ models of renal cilia, presumably by disrupting either the diffusion barrier or vesicular trafficking, either of which would perturb ciliary assembly. How they are specifically targeted to the base of the cilium remains elusive: there are no obvious ciliary targeting motifs in the ion channels (e.g. binding sites for X-box or the Fox-J1 transcription factors). However, there is a di-arginine endoplasmic reticulum retrieval and retention motif in all four of these ion channels, which is common in transmembrane proteins mutated in ciliopathies, such as TMEM237 and TMEM67. For example, frequent and recurrent pathogenic TMEM67 mutations (OMIM 609884; Arg440Gln, Arg441Leu, Arg441Cys, Arg549Cys) all cause a missense change of one of the arginine residues in this motif. Given that all of these transmembrane proteins will be packaged and transported as vesicles, it is possible that they are sent specifically to the ciliary base or ciliary pocket, although live-cell imaging of fluorescently labeled proteins would be required to answer that question.

The ciliogenesis defect seen in 3D spheroids after knockdown of these ion channels can be rescued by exogenous overexpression of the WT human ion channels, which are not targeted by the mouse siRNA. However, overexpression of mutated versions of KCNQ1 failed to rescue the ciliogenesis defect after knockdown, suggesting that these mutations affect protein structure or function in such a way that is crucial to the formation of renal primary cilia. These $K C N Q 1$ mutations are found in patients with inherited diseases encompassing ciliopathy-like phenotypes including congenital heart disease and sensorineural hearing loss. The characterization of the function of this ion channel in primary cilia, with an additional role in ciliogenesis, provides novel insights into disease pathogenesis in these patients. In contrast to our findings, a functional genomic screen for modulators of ciliogenesis and cilium length in retinal pigment epithelial cells did not identify these four ion channels (Kim et al., 2010). However, we have ascertained that these same ion channels are not expressed in this cell type [RNA sequence data deposited, SRA study PRJNA298886 'ciliated cell lines', accession SRP064956; data accession numbers: SRX1353143, SRX1411364, SRX1411444, SRX1411451, SRX1411453 and SRX1411455 (website http:// trace.ncbi.nlm.nih.gov/Traces/sra/)]. We therefore conclude that the data from Kim et al. neither support nor contradict our findings, but complement each other. Furthermore, it would be interesting to use antagonists to block these channels pharmacologically to determine the effect on cilia.

Mutations in a range of cilia proteins lead to a suite of diseases known as the ciliopathies. Proteins mutated in ciliopathies include structural proteins and proteins involved in ciliary signaling (Sang et al., 2011), including ion channels. For example, PKD1 and $P K D 2$, which encode the polycystins that heterodimerize to form a $\mathrm{Ca}^{2+}$ ion channel, are mutated in polycystic kidney disease (PKD) (Barr et al., 2001). Mutations in PKD1 or PKD2 can lead to disturbed ciliary signaling without affecting cilium structure (Nauli et al., 2003; Low et al., 2006). Our findings suggest that damaging mutations in $K C N Q 1, K C N J 10, K C N F 1$ and CLCN4 cause defects in cilia structure, but also that mutations affecting the ion channel function might not affect the cilia (e.g. KCNJ10). These findings not only suggest a possible role for primary cilia in the pathophysiology of conditions associated with LQTS, but they offer new insights into the general importance of ion channels and ion signaling in ciliopathies. Identification of four new ion channels that localize to primary cilia and are required for ciliogenesis, supports previously published data showing that the length of the ciliary structure is regulated by ion current, and ion channel stimulation can result in cilia-generated signaling (Ludington et al., 2015). Our data suggest that disturbance of ion balance by loss of the ion channel KCNQ1 results in ciliopathy phenotypes in vitro. By contrast, our data also suggest that KCNF1 and CLCN4 may be new candidates in human ciliopathies. However, KCNJ10-associated EAST syndrome does not apparently involve ciliogenesis defects.

The involvement of ion channels in disease, particularly heart disease, has been appreciated for over two decades (Ackerman and Clapham, 1997); however, the role of ciliary ion channels in this disease process is not entirely understood. Whereas the general 


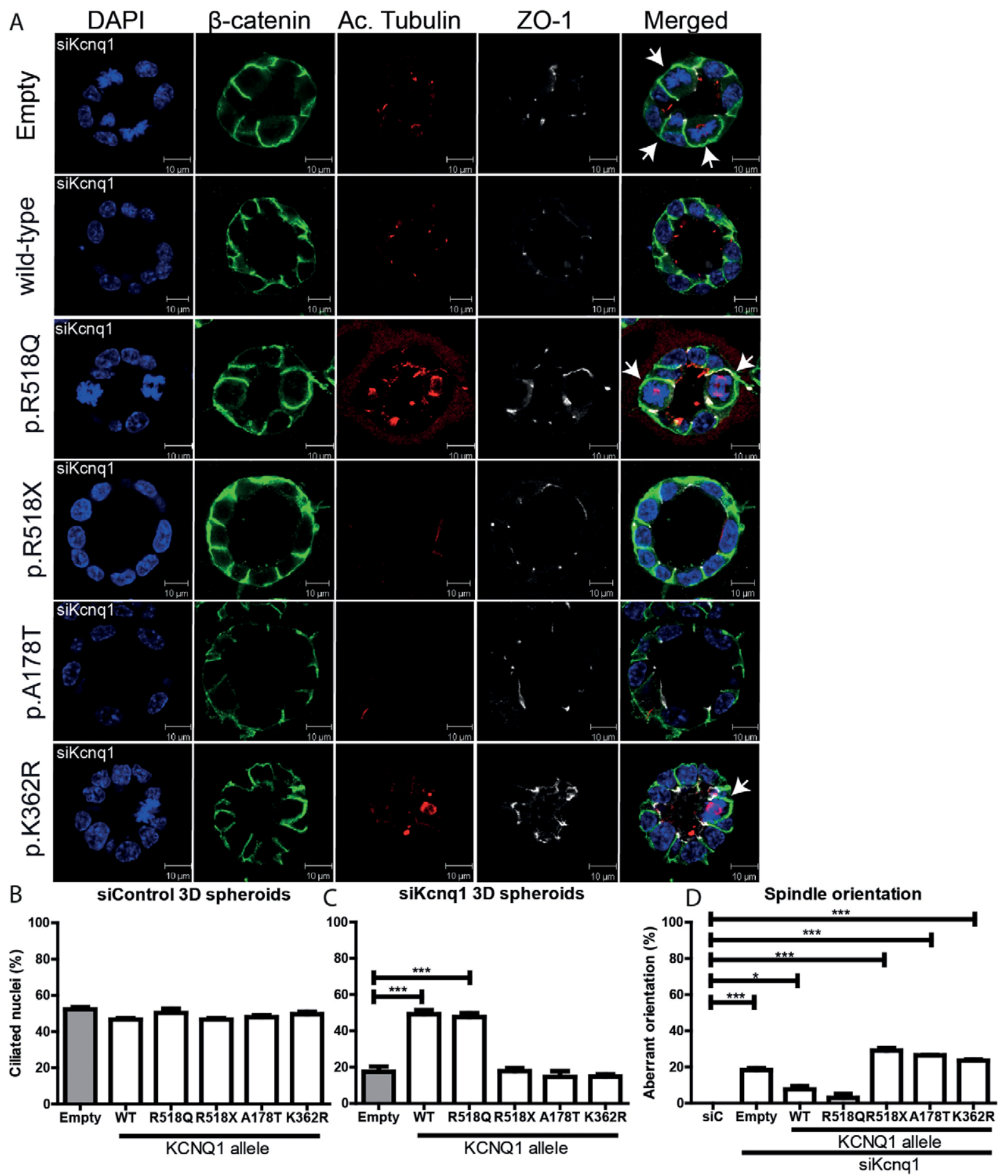

Fig. 5. LQTS-associated KCNQ1 mutant alleles in 3D spheroids. (A) Immunostaining of spheroids of mIMCD3 cells for cilia (acetylated $\alpha$-tubulin, red), tight junctions (ZO-1, white), and adherens junctions ( $\beta$-catenin, green) with DAPI counterstaining (blue) showing loss of cilia after Kcnq1 siRNA transfection (20 nM), and rescue of ciliation by wild-type (WT) and p.R518Q KCNQ1. No rescue of cilia is observed after overexpression of p.R518X, p.A178T and p.K362R KCNQ1. (B,C) Quantification (mean \pm s.e.m., \%) of ciliary frequency in spheroids shows loss of cilia in spheroids depleted for Kcnq1, and restoration of ciliary frequency was obtained upon transfection with WT KCNQ1. Overexpression of variant KCNQ1 did not affect ciliation in siControl-transfected cells. In siKcnq1-transfected cells, overexpression of KCNQ1 p.R518Q also rescues ciliation, but p.R518X, p.A178T and p.K362R KCNQ1 do not restore cilia. 20 spheroids were scored per condition ( $n=3$ ). (D) Aberrant spindle formation was observed in cells siRNA-depleted for Kcnq1 and overexpressing KCNQ1 mutant alleles (white arrows, panel A). 20 spheroids were scored per condition $(n=3) .{ }^{*} P<0.05,{ }^{* * *} P<0.001$ (one-way ANOVA with Dunnett's test).

importance of primary cilia at the embryonic node is understood to regulate normal heart laterality (Slough et al., 2008), the role of ciliary ion channels in this process has not been examined. Recently, a central role for cilia in congenital heart disease (CHD) has been identified by a recessive forward genetic screen in mice, and it could be inferred that, to some extent, ion channels in cilia might account for such phenotypes ( $\mathrm{Li}$ et al., 2015). Whole-exome sequencing in that study revealed that of 61 genes mutated in CHD, 34 encoded 
cilia-related proteins and 16 encoded proteins involved in cilia signaling ( $\mathrm{Li}$ et al., 2015). In addition to this, a number of ciliopathies, including Ellis van Creveld syndrome (MIM 225500) and Alström syndrome (MIM 203800), can include CHD in their phenotypic spectrum (Baker and Beales, 2009). Taken together, these findings suggest that ciliary ion channels are important for normal heart development and function, and that KCNQ1associated heart disease might involve cilia dysfunction.

Although highly speculative, our findings suggest that LQTS patients with $K C N Q 1$ mutations might possibly harbor a subclinical renal phenotype (most likely a mild nephronophthisis); acquiring renal sonograms and/or urine concentration data from patients with KCNQ1 mutations would be an interesting follow-up to this study. Additionally, analyzing whole-exome sequencing data from patient cohorts with end-stage renal disease or renal ciliopathies might uncover enrichment for $K C N Q 1$ variants or mutations. Interestingly, in patients with renal failure, hypertension and many other factors can contribute to consequent cardiovascular burden and acquired LQTS (Gussak and Gussak, 2007). Our data might likewise point to the dysfunction of cilia as one of the factors contributing to acquired LQTS.

Ciliopathies are defined as genetic diseases involving defects in proteins localizing to the cilium or associated complexes and pathways. Ciliary perturbation is not limited to cilia structure, but also function, including signaling through ion channels. This makes careful classification and diagnosis on phenotypic, genotypic and finally on physiological grounds challenging but nonetheless important (Baker and Beales, 2009). Ciliopathies cover a wide range of genotypes and phenotypes, and the list of ciliopathies is ever expanding. Our data elucidating the function of four new primary cilia ion channels suggest that KCNQ1 could be implicated in renal ciliopathies, but, in contrast, that $\mathrm{KCNJ} 10$ is not. Furthermore, KCNF1 and CLCN4 could be unidentified genetic causes of ciliopathies involving renal defects. These new insights into the pathophysiological disease mechanisms of patients with KCNQ1 mutations will inspire future research concerning therapeutic approaches and molecular genetic screening of ion channel genes in ciliopathy patients.

\section{MATERIALS AND METHODS \\ Cell culture}

Mouse inner medullary collecting duct (mIMCD3) cells were cultured as previously described (Slaats et al., 2014). Human fibroblasts were grown from skin biopsies in Dulbecco's modified Eagle's medium (DMEM) supplemented with $10 \%$ fetal calf serum (FCS) and $1 \%$ penicillinstreptomycin. Collection of human fibroblast samples was approved by the Medical Ethics Committee of the University Medical Center Utrecht. Informed consent was obtained for all tissue donors; all clinical investigation have been conducted according to the principles expressed in the Declaration of Helsinki. Cells were incubated at $37^{\circ} \mathrm{C}$ in $5 \% \mathrm{CO}_{2}$ to $\sim 90 \%$ confluence. Fibroblasts were serum starved for $48 \mathrm{~h}$ prior to fixation.

\section{Reverse genetics visual screen and data analysis}

The reverse genetics visual screen was performed as described previously (Wheway et al., 2015). In brief, pools of four Dharmacon siGENOME small interfering RNAs (siRNAs; final total concentration $50 \mathrm{nM}$ ) were used to silence gene expression of 340 mouse ion channel genes in mIMCD3 cells (Table S1). siRNAs were dispensed into 96-well optical bottom View Plates (Perkin Elmer) using the Bravo liquid handling platform, Lipofectamine RNAiMAX (Life Technologies) in suspension in OptiMEM was added to the wells' FluidX dispenser and, after $20 \mathrm{~min}$, cells in a suspension of OptiMEM were added to the wells, also using a FluidX dispenser. All knockdowns were performed in duplicate to produce two independent replicates. Cells were fixed in methanol and immunostained with a monoclonal antibody against acetylated $\alpha$-tubulin (clone 6-11-B1, catalogue number T6793, lot no 070M4755, Sigma-Aldrich), and DAPI and TOTO3, at $72 \mathrm{~h}$ post-transfection and imaged within $48 \mathrm{~h}$ of immunostaining. Images from six fields of view per well were captured from three focal planes using a $\times 40$ air objective lens on a PerkinElmer Operetta automated imaging platform. siRNA pools targeting Ift $88, M \mathrm{ks} I$ and Rpgripll were used as positive controls for an effect on ciliogenesis (Fig. 1B). Plkl was used as a positive control for effect on cell number and as a measure of the efficiency of transfection. Non-targeting siRNAs and siRNA targeting human MLNR (which does not target any mouse gene) were used as negative controls. Using this system, cilia are observed as discrete dots above the nuclei (Wheway et al., 2015). The dots were recognized using the Perkin-Elmer 'find spots' algorithm during image analysis. The percentage of cells with a single cilium was then calculated for each experimental sample, and $z$ scores for this value were then calculated from the negative controls. The $z$ scores were calculated for each batch of 10 plates, which were all processed simultaneously for staining and imaging, to minimize variability. Each plate contained eight positive and eight negative controls.

\section{Antibodies and reagents}

Antibodies used were rabbit anti-CLCN4 (Abcam, 105144), rabbit antiKCNF1 (Bioss bs12173R), rabbit anti-KCNQ1 (Alomone labs, APC-022), rabbit anti-KCNJ10 (Alomone labs, APC-035), guinea pig anti-RPGRIP1L (Arts et al., 2007), mouse anti-acetylated tubulin (clone 6-11-B1, SigmaAldrich, T6793) and mouse anti- $\beta$-actin (clone AC-15, Sigma-Aldrich, A5441), rat anti-ZO-1 (Santa Cruz Biotechnology) and rabbit anti- $\beta$-catenin (BD Bioscience) antibodies.

Plasmid DNA transfection was performed with Lipofectamine 2000 (Invitrogen, 11668-019), according to the supplier's protocol. Opti-MEM (Invitrogen, 31985-062) was used to dilute the plasmids KCNJ10::C-GFP (Origene RG213543), KCNQ1 and CLCN4::N-GFP, or mutant alleles.

Lipofectamine RNAimax (Invitrogen, 13778-075) was used for siRNA transfection at a total final concentration of $20 \mathrm{nM}$, according to the supplier's protocol. Opti-MEM (Invitrogen, 31985-062) was used to dilute the ON-TARGETplus siRNA SMARTpools (Thermo Scientific Dharmacon) [non-targeting pool, 5'-GGUUUACAUGUCGACUAA-3', 5'-UGGUUUACAUGUUGUGUGA-3', 5'-UGGUUUACAUGUUUUCUGA-3' and 5'-UGGUUUACAUGUUUUCCUA-3' (D-00181010); mouse Ift $88,5^{\prime}$-CGGAGAAUGUUGAAUGUUU-3', 5'-GCUUGGAGCUUAUUACAUU-3', 5'-CGUCAGCUCUCACUAAUAA-3' and 5'-GUAGCUAGCUGCUUUAGAA-3' (L-050417-00); mouse Kcnq1, 5'-GCAGCGCGGUGGUCAAGAA-3', 5'-GAUAGGAGGCCAGACCAUU5'-GGAAGUGUUUCGUGUACCA-3' and 5'-GAUGAGUCCUGGAGAGAAG-3' (L-046886-00); mouse Clcn4-2, 5'-GAGCUAAUCUUGGCUAUAA-3', 5'-GGAAAUGGACUCUUCUAAU-3', 5'-UAGAGGAGGUCAGUUACUA-3' and 5'-UGACUCGGCCUGUGGAUGA-3' (L-062747-00); mouse Kcnf1 5'. GCGAAGACAUUGAGAUCGU-3'， 5'-ACACAGCGAUACCUUCAUU-3', 5'-CAAGCGCAGUUUCAAGGAA-3' and 5'-UGAUGGAGCUGACCAACGU-3' (L-056002-00); and mouse Kcnj10 5'GCACAUUGCUGACAAACGU-3', 5'-GAAAUCUUCAUCACAGGUA-3', 5'-GCAAUGGCGCUACAAGCUU-3' and 5'-GCAAAUACAUAGCUGACUU-3' (L-042680-00)] to $20 \mathrm{nM}$.

\section{RT-qPCR}

RNA was isolated, cDNA was synthesized and RT-qPCR analysis was performed as previously described (Slaats et al., 2014). The mouse primer sequences (Sigma) used and concomitant annealing temperatures are: Kcnq1, Fw 5'-CAAAGACCGTGGCAGTAAC-3' and Rv 5'-CCTTCATTGCTGGCTACAAC- $3^{\prime}\left(55^{\circ} \mathrm{C}\right)$; Kcnfl, Fw $5^{\prime}$-CGTGGCAGGCGAAGACATT-3' and Rv 5'-CCCCCGCCAAACAGTTGAT-3' (60 $\left.{ }^{\circ} \mathrm{C}\right)$; Kcnj10, Fw 5'-CAGGTCAAATGTATTTAG-3' and Rv 5'-CAAGAGATBCATCCAGTTTATTT- $3^{\prime}\left(61^{\circ} \mathrm{C}\right)$; Clcn 4 , Fw 5'-GCGTCTCATCGGGTTTGC-3 and Rv 5'-TTGCCACAATGCCCTCTTG-3' $\left(55^{\circ} \mathrm{C}\right)$; and Rpl27, Fw 5'-CGCCCTCCTTTCCTTTCTGC-3' and Rv 5'-GGTGCCATCGTCAA TGTTCTTC- $3^{\prime}\left(53^{\circ} \mathrm{C}\right)$. The $\Delta \Delta \mathrm{CT}$ method was used for statistical analysis to determine gene expression levels. 


\section{In vitro mutagenesis}

KCNJ10 mutations were introduced through site-directed mutagenesis. Presence of the mutation in the plasmid was verified by Sanger sequencing (all primers are available upon request)

\section{Immunofluorescence}

For immunostaining, mIMCD3 cells were grown on coverslips and fixed for $5 \mathrm{~min}$ in ice-cold methanol and blocked $60 \mathrm{~min}$ in $1 \%$ bovine serum albumin (BSA). Primary antibody incubations (mouse anti-acetylated tubulin 1:20,000; rabbit anti-CLCN4, -KCNF1, -KCNQ1 or -KCNJ10, 1:200) were performed at $4{ }^{\circ} \mathrm{C}$ overnight in $1 \%$ BSA. Goat anti-mouse-IgG conjugated to Alexa Fluor 488 or anti-rabbit-IgG conjugated to Alexa Fluor 568 secondary antibody (Invitrogen, dilution 1:500) and Hoechst 333 incubations were performed for $2 \mathrm{~h}$ at room temperature. Coverslips were mounted in Fluormount G (Cell Lab, Beckman Coulter). Confocal imaging was performed using Zeiss LSM700 confocal laser microscope and images were processed with the ZEN 2012 software. 3D spheroid Matrigel assays with mIMCD3 cells were performed as previously described (Giles et al., 2014). Spindle orientation was determined in cells in metaphase, anaphase or telophase; serial $z$-stack images were taken of each spheroid (typically every $1 \mu \mathrm{m}$ ). If one of the spindle poles protruded into the lumen of the spheroid, the angle of the spindle orientation (line between two spindle poles) with respect to the radius is measured (ideally, $90^{\circ}$ ). Angle deviations of greater than $11^{\circ}$ perpendicular to the spheroid radius will be scored as misoriented (Luijten et al., 2013).

\section{Western blotting}

Protein lysates were prepared using RIPA lysis buffer. To correct for protein content, a BCA protein assay (Pierce) was performed. $\beta$-actin (antibody at 1:15,000) was used as loading control in combination with Coomassie Blue staining. After SDS-PAGE separation and transfer, the PVDF membranes were blocked in 5\% dried low-fat milk in TBS with $0.5 \%$ Tween 20 . The primary antibodies rabbit anti-CLCN4 (1:500), rabbit anti-KCNQ1 (1:300), rabbit anti-KCNF1 (1:500), rabbit anti-KCNJ10 (1:500), rabbit anti-GFP $(1: 1000)$ antibodies were incubated overnight at $4^{\circ} \mathrm{C}$. The secondary swine anti-rabbit-IgG and rabbit anti-mouse-IgG antibodies, which were conjugated to horseradsih peroxidase (HRP; DAKO, dilution 1:2000), were incubated for $1 \mathrm{~h}$ at room temperature. The ECL Chemiluminescent Peroxidase Substrate kit (Sigma, CPS1120-1KT) was used for development. Scans of the blots were made with the BioRad ChemiDoc XRS+ device with Image Lab software 4.0.

\section{Histology}

Kidney tissue sections of healthy Lewis rats embedded in paraffin were deparaffinized and incubated at $100^{\circ} \mathrm{C}$ in citrate- $\mathrm{HCl}$ buffer for $15 \mathrm{~min}$ (citrate$\mathrm{HCl}$ was skipped for rabbit anti-KCNF1 antibody staining). Sections were blocked using peroxidase block for $15 \mathrm{~min}$ followed by $5 \%$ normal goat serum (NGS) block for $30 \mathrm{~min}$. The sections were stained with rabbit anti-KCNJ10, rabbit anti-KCNQ1 (1:200), rabbit anti-CLCN4 (1:300) and rabbit-antiKCNF1 (1:50) antibodies in 1\% normal goat serum (NGS) for $1 \mathrm{~h}$ at room temperature. Finally, BrightVision Poly HRP goat anti-rabbit-IgG antibody (Immunologic, DPVR55HRP) was incubated for $30 \mathrm{~min}$ at room temperature. NovaRED substrate kit for Peroxidase (Vector, SK-4800) was used and counterstained with hematoxylin. Images were taken using a $40 \times$ objective.

\section{FACS}

To quantify cell cycle phase distribution, cells were incubated with $10 \mu \mathrm{M}$ BrdU for $30 \mathrm{~min}$ and fixed in ice-cold $70 \%$ ethanol. Samples were stained for FACS analysis with BrdU mouse monoclonal antibody conjugated to Alexa Fluor 647 (1:200; Invitrogen) in $0.1 \%$ BSA in PBS-T (PBS with $0.5 \%$ Tween 20) for $1 \mathrm{~h}$ on ice, and DAPI in PBS. 10,000 events were measured with a BD FACSCanto II flow cytometer and analyzed using BD FACSDiva Software.

\section{Statistics}

The effect of siRNA knockdown on ciliogenesis in the screen was assessed by calculating the percentage of cells with a single cilium, with the statistical significance of this effect assessed by calculating $z$ scores $\left(z_{\text {cilia }}\right) . z$ scores were also calculated for the effect of the knockdown on cell number $\left(z_{\text {cell }}\right)$, to enable the exclusion of any effects of cell proliferation or apoptosis on ciliogenesis given that these could be due to non-specific secondary processes. We considered a siRNA to have a significant effect on ciliogenesis when it had a $z_{\text {cilia }}<-2$ and $z_{\text {cell }} \geq-2$ in both independent experimental replicates.

In the follow-up experiments, $P$-values were calculated for normally distributed data sets using a two-tailed Student's $t$-test, one-way ANOVA with Dunnett's post-hoc test, or two-way ANOVA with Bonferroni post-hoc tests. Statistical analyses represent the mean of at least three independent experiments; error bars represent s.e.m. or as otherwise indicated.

\section{Acknowledgements}

We thank Sebastiaan Noordegraaf voor for help with RT-qPCR, Glenn van de Hoek and Ka Man Wu for help with in vitro mutagenesis, Jaap Joles for rat kidney sections, as well as Oliver Blacque (Dublin) and Joost Hoenderop and Erik de Vrieze (Nijmegen) for experiments not included in the manuscript. The Cell Microscopy Center and the Flow Cytometry Core Facility at the UMC Utrecht provided expert services. CLCN4 pcDNA6.2-N-GFP was a kind gift from Erica Davis (Duke University, Durham, USA), and wild-type and variant KCNQ1 plasmids were a kind gift from Marcel van der Heyden and Paul de Bakker (Utrecht University, Utrecht, The Netherlands).

\section{Competing interests}

The authors declare no competing or financial interests.

\section{Author contributions}

G.G.S., G.W., C.A.J. and R.H.G. designed the experiments. G.G.S., G.W., V.F., K.S., I.L., K.D.O. and R.H.G. performed the experiments. G.G.S., G.W., C.A.J. and R.H.G. analyzed the data. B.W.M.B., M.G.K.-V. and M.R.L. contributed reagents, materials and/or analysis tools. G.G.S., G.W., N.V.K., C.A.J. and R.H.G. wrote the paper.

\section{Funding}

This work was supported by the European Union FP7/2009 Consortium 'SYSCILIA' [grant number 241955 to R.H.G., C.A.J.]; the Dutch Kidney Foundation 'KOUNCIL' [grant number CP11.18 to N.V.K., M.R.L., R.H.G.]; and by the Medical Research Council [grant number MR/M000532/1 to C.A.J.]. Deposited in PMC for release after 6 months.

\section{Supplementary information}

Supplementary information available online at

http://jcs.biologists.org/lookup/suppl/doi:10.1242/jcs.176065/-/DC1

\section{References}

Ackerman, M. J. and Clapham, D. E. (1997). Ion channels-basic science and clinical disease. N. Engl. J. Med. 336, 1575-1586.

Arts, H. H., Doherty, D., van Beersum, S. E. C., Parisi, M. A., Letteboer, S. J. F., Gorden, N. T., Peters, T. A., Märker, T., Voesenek, K., Kartono, A. et al. (2007). Mutations in the gene encoding the basal body protein RPGRIP1L, a nephrocystin-4 interactor, cause Joubert syndrome. Nat. Genet. 39, 882-888.

Baker, K. and Beales, P. L. (2009). Making sense of cilia in disease: the human ciliopathies. Am. J. Med. Genet. C Semin. Med. Genet. 151C, 281-295.

Barr, M. M., DeModena, J., Braun, D., Nguyen, C. Q., Hall, D. H. and Sternberg, P. W. (2001). The Caenorhabditis elegans autosomal dominant polycystic kidney disease gene homologs lov-1 and pkd-2 act in the same pathway. Curr. Biol. 11, 1341-1346.

Basten, S. G. and Giles, R. H. (2013). Functional aspects of primary cilia in signaling, cell cycle and tumorigenesis. Cilia 2, 6 .

Bellocq, C., van Ginneken, A. C. G., Bezzina, C. R., Alders, M., Escande, D., Mannens, M. M. A. M., Baro, I. and Wilde, A. A. M. (2004). Mutation in the KCNQ1 gene leading to the short QT-interval syndrome. Circulation 109 2394-2397.

Chen, Y.-H., Xu, S.-J., Bendahhou, S., Wang, X.-L., Wang, Y., Xu, W.-Y., Jin H.-W., Sun, H., Su, X.-Y., Zhuang, Q.-N. et al. (2003). KCNQ1 gain-of-function mutation in familial atrial fibrillation. Science 299, 251-254.

D'Angelo, A. and Franco, B. (2011). The primary cilium in different tissues-lessons from patients and animal models. Pediatr. Nephrol. 26, 655-662.

Giles, R. H., Ajzenberg, H. and Jackson, P. K. (2014). 3D spheroid model of mIMCD3 cells for studying ciliopathies and renal epithelial disorders. Nat. Protoc. 9, 2725-2731.

Gussak, I. and Gussak, H. M. (2007). Sudden cardiac death in nephrology: focus on acquired long QT syndrome. Nephrol. Dial Transplant. 22, 12-14. 
Hu, Q., Milenkovic, L., Jin, H., Scott, M. P., Nachury, M. V., Spiliotis, E. T. and Nelson, W. J. (2010). A septin diffusion barrier at the base of the primary cilium maintains ciliary membrane protein distribution. Science $\mathbf{3 2 9}, 436-439$.

Jespersen, T., Rasmussen, H. B., Grunnet, M., Jensen, H. S., Angelo, K., Dupuis, D. S., Vogel, L. K., Jorgensen, N. K., Klaerke, D. A. and Olesen, S.-P (2004). Basolateral localisation of KCNQ1 potassium channels in MDCK cells: molecular identification of an N-terminal targeting motif. J. Cell Sci. 117, 4517-4526.

Kapplinger, J. D., Tester, D. J., Salisbury, B. A., Carr, J. L., Harris-Kerr, C., Pollevick, G. D., Wilde, A. A. M. and Ackerman, M. J. (2009). Spectrum and prevalence of mutations from the first 2,500 consecutive unrelated patients referred for the FAMILION ${ }^{\circledR}$ long QT syndrome genetic test. Heart Rhythm 6 1297-1303

Kim, J., Lee, J. E., Heynen-Genel, S., Suyama, E., Ono, K., Lee, K., Ideker, T., Aza-Blanc, P. and Gleeson, J. G. (2010). Functional genomic screen for modulators of ciliogenesis and cilium length. Nature 464, 1048-1051.

Lee, M. P., Hu, R.-J., Johnson, L. A. and Feinberg, A. P. (1997). Human KVLQT1 gene shows tissue-specific imprinting and encompasses Beckwith-Wiedemann syndrome chromosomal rearrangements. Nat. Genet. 15, 181-185.

Li, Y., Klena, N. T., Gabriel, G. C., Liu, X., Kim, A. J., Lemke, K., Chen, Y., Chatterjee, B., Devine, W., Damerla, R. R. et al. (2015). Global genetic analysis in mice unveils central role for cilia in congenital heart disease. Nature 521, 520-524.

Loudon, K. W. and Fry, A. C. (2014). The renal channelopathies. Ann. Clin Biochem. 51, 441-458.

Low, S. H., Vasanth, S., Larson, C. H., Mukherjee, S., Sharma, N., Kinter, M. T., Kane, M. E., Obara, T. and Weimbs, T. (2006). Polycystin-1, STAT6, and P100 function in a pathway that transduces ciliary mechanosensation and is activated in polycystic kidney disease. Dev. Cell 10, 57-69.

Lu, Q., Insinna, C., Ott, C., Stauffer, J., Pintado, P. A., Rahajeng, J., Baxa, U., Walia, V., Cuenca, A., Hwang, Y.-S. et al. (2015). Early steps in primary cilium assembly require EHD1/EHD3-dependent ciliary vesicle formation. Nat. Cell Biol. $17,531$.

Ludington, W. B., Ishikawa, H., Serebrenik, Y. V., Ritter, A., Hernandez-Lopez, R. A., Gunzenhauser, J., Kannegaard, E. and Marshall, W. F. (2015). A systematic comparison of mathematical models for inherent measurement of ciliary length: how a cell can measure length and volume. Biophys. J. 108 1361-1379

Luijten, M. N. H., Basten, S. G., Claessens, T., Vernooij, M., Scott, C. L., Janssen, R., Easton, J. A., Kamps, M. A. F., Vreeburg, M., Broers, J. L. V. et al. (2013). Birt-Hogg-Dube syndrome is a novel ciliopathy. Hum. Mol. Genet. 22 4383-4397.

Nauli, S. M., Alenghat, F. J., Luo, Y., Williams, E., Vassilev, P., Li, X., Elia, A. E. H., Lu, W., Brown, E. M., Quinn, S. J. et al. (2003). Polycystins 1 and 2 mediate mechanosensation in the primary cilium of kidney cells. Nat. Genet. 33 129-137

Neyroud, N., Tesson, F., Denjoy, I., Leibovici, M., Donger, C., Barhanin, J., Fauré, S., Gary, F., Coumel, P., Petit, C. et al. (1997). A novel mutation in the potassium channel gene KVLQT1 causes the Jervell and Lange-Nielsen cardioauditory syndrome. Nat. Genet. 15, 186-189.

Oh, E. C. and Katsanis, N. (2012). Cilia in vertebrate development and disease. Development 139, 443-448.

Parrock, S., Hussain, S., Issler, N., Differ, A.-M., Lench, N., Guarino, S. Oosterveld, M. J. S., Keijzer-Veen, M., Brilstra, E., van Wieringen, H. et al.
(2013). KCNJ10 mutations display differential sensitivity to heteromerisation with KCNJ16. Nephron Physiol. 123, 7-14

Pazour, G. J., Dickert, B. L., Vucica, Y., Seeley, E. S., Rosenbaum, J. L., Witman G. B. and Cole, D. G. (2000). Chlamydomonas IFT88 and its mouse homologue, polycystic kidney disease gene $\operatorname{tg} 737$, are required for assembly of cilia and flagella. J. Cell Biol. 151, 709-718.

Refsgaard, L., Holst, A. G., Sadjadieh, G., Haunso, S., Nielsen, J. B. and Olesen, M. S. (2012). High prevalence of genetic variants previously associated with LQT syndrome in new exome data. Eur. J. Hum. Genet. 20, 905-908.

Reichold, M., Zdebik, A. A., Lieberer, E., Rapedius, M., Schmidt, K., Bandulik, S., Sterner, C., Tegtmeier, I., Penton, D., Baukrowitz, T. et al. (2010). KCNJ10 gene mutations causing EAST syndrome (epilepsy, ataxia, sensorineural deafness, and tubulopathy) disrupt channel function. Proc. Natl. Acad. Sci. USA 107, 14490-14495.

Sang, L., Miller, J. J., Corbit, K. C., Giles, R. H., Brauer, M. J., Otto, E. A., Baye, L. M., Wen, X., Scales, S. J., Kwong, M. et al. (2011). Mapping the NPHP-JBTS MKS protein network reveals ciliopathy disease genes and pathways. Cell 145 513-528.

Sanguinetti, M. C., Curran, M. E., Zou, A., Shen, J., Spector, P. S., Atkinson, D. L. and Keating, M. T. (1996). Coassembly of K(V)LQT1 and minK (IsK) proteins to form cardiac I(Ks) potassium channel. Nature 384, 80-83.

Satir, P. and Christensen, S. T. (2008). Structure and function of mammalian cilia. Histochem. Cell Biol. 129, 687-693.

Scheel, O., Zdebik, A. A., Lourdel, S. and Jentsch, T. J. (2005). Voltagedependent electrogenic chloride/proton exchange by endosomal CLC proteins. Nature 436, 424-427.

Slaats, G. G., Ghosh, A. K., Falke, L. L., Le Corre, S., Shaltiel, I. A., van de Hoek, G., Klasson, T. D., Stokman, M. F., Logister, I., Verhaar, M. C. et al. (2014) Nephronophthisis-associated CEP164 regulates cell cycle progression, apoptosis and epithelial-to-mesenchymal transition. PLoS Genet. 10, e1004594

Slough, J., Cooney, L. and Brueckner, M. (2008). Monocilia in the embryonic mouse heart suggest a direct role for cilia in cardiac morphogenesis. Dev. Dyn. 237, 2304-2314.

Stattin, E.-L., Boström, I. M., Winbo, A., Cederquist, K., Jonasson, J., Jonsson, B.-A., Diamant, U.-B., Jensen, S. M., Rydberg, A. and Norberg, A. (2012). Founder mutations characterise the mutation panorama in 200 Swedish index cases referred for Long QT syndrome genetic testing. BMC Cardiovasc. Disord. 12, 95

Tanaka, T., Nagai, R., Tomoike, H., Takata, S., Yano, K., Yabuta, K., Haneda, N. Nakano, O., Shibata, A., Sawayama, T. et al. (1997). Four novel KVLQT1 and four novel HERG mutations in familial long-QT syndrome. Circulation 95, 565-567. Tester, D. J., Will, M. L., Haglund, C. M. and Ackerman, M. J. (2005). Compendium of cardiac channel mutations in 541 consecutive unrelated patients referred for long QT syndrome genetic testing. Heart Rhythm 2, 507-517. Wang, Q., Curran, M. E., Splawski, I., Burn, T. C., Millholland, J. M., VanRaay, T. J., Shen, J., Timothy, K. W., Vincent, G. M., de Jager, T. et al. (1996) Positional cloning of a novel potassium channel gene: KVLQT1 mutations cause cardiac arrhythmias. Nat. Genet. 12, 17-23

Wheway, G., Schmidts, M., Mans, D. A., Szymanska, K., Nguyen, T.-M. T. Racher, H., Phelps, I. G., Toedt, G., Kennedy, J., Wunderlich, K. A. et al. (2015). An siRNA-based functional genomics screen for the identification of regulators of ciliogenesis and ciliopathy genes. Nat. Cell Biol. 17, 1074-1087.

Wilson, P. D. (2004). Polycystic kidney disease. N. Engl. J. Med. 350, 151-164. 